\title{
Clinical outcomes of two revision strategies for failed total disc replacements
}

\author{
Ilona Punt $\cdot$ Paul Willems $\cdot$ Steven Kurtz • \\ Lodewijk van Rhijn $\cdot$ André van Ooij
}

Received: 30 March 2011/Revised: 15 April 2012/Accepted: 25 April 2012/Published online: 11 May 2012

(C) The Author(s) 2012. This article is published with open access at Springerlink.com

\begin{abstract}
Purpose To compare mid-term clinical outcomes of two revision strategies for patients with failed SB Charité III total disc replacements (TDRs).

Methods Eighteen patients with a failed TDR underwent posterolateral instrumented fusion (fusion group); in 21 patients, the TDR was removed and the intervertebral defect was filled with a bone strut graft, followed by an instrumented posterolateral fusion (removal group). Visual analogue scale (VAS) for pain and Oswestry Disability Index (ODI) were completed pre- and post-revision surgery. Intra- and post-operative complications of both revision strategies were assessed.

Results Mean follow-up was 3.7 years (range 1.0-6.4) in the removal group and 4.4 years (range 0.7-11.0) in the fusion group. Although the removal group showed a significantly lower VAS and ODI score post-revision surgery as compared to preoperative $(P<0.01$ and $P=0.01$, respectively), no significant differences were found between the removal and fusion groups before and after
\end{abstract}

I. Punt $(\bowtie) \cdot$ P. Willems $\cdot$ L. van Rhijn

Department of Orthopaedic Surgery, Maastricht University

Medical Center, P.O. Box 5800, 6202 AZ Maastricht,

The Netherlands

e-mail: i.punt@mumc.nl

I. Punt · P. Willems · L. van Rhijn

Research School CAPHRI, Maastricht University, P.O. Box 616, 6200 MD Maastricht, The Netherlands

S. Kurtz

Drexel University, Philadelphia, PA, USA

A. van Ooij

Department of Orthopaedic Surgery, Viecuri Medical Centre,

P.O. Box 1926, 5900 BX Venlo, The Netherlands revision surgery in VAS and ODI. A clinical relevant improvement in VAS and ODI was found in 47 and $21 \%$ respectively in the removal group, and in 22 and $27 \%$ respectively in the fusion group. Substantial complications were observed only in the removal group.

Conclusions Both procedures showed improvement clinically. There were no significant additional benefits of removing the TDR as compared to fusion alone at midterm follow-up. The clinical decision to remove the TDR should be carefully weighed up against potential risks and complications of this procedure.

Keywords Total disc replacement - Spinal fusion . Revision surgery · VAS · Oswestry

\section{Introduction}

Lumbar total disc replacement (TDR) is increasingly used in the surgical treatment of degenerative disc disease (DDD). TDR aims to remove the pain source while preserving vertebral motion at the degenerative operated level(s) to prevent the development of adjacent segment degeneration [1-6]. The debate whether TDR is more effective than lumbar spinal fusion in treating DDD is still going on [5, 7-12]. Recently, a prospective randomized study showed that there are no differences in safety and clinical outcomes after TDR as compared to spinal fusion, at a follow-up period of 5 years [8]. It was further reported that between 2 and 5-years follow-up, only in the TDR group device failures had been observed [8].

Potential complications after TDR are recurrent back and leg pain, caused by facet joint degeneration, subsidence, polyethylene wear, migration and adjacent segment degeneration $[5,7,10,13]$. This warrants the need for 
surgical revision strategies [14]. In a recent systematic review of Eerenbeemt et al. [11], an overall revision surgery rate ranging from 3.7 to $11.4 \%$ was found after TDR. An important question we should ask ourselves is: Will revision surgery be beneficial, and if so, what would be the best revision strategy for failed TDR? In a previous study, we reported short-term results of two revision strategies with a follow-up of 1 year, showing that TDR removal gave better results than posterolateral instrumented fusion alone [15]. Larger groups and longer follow-up were thought to be necessary to support possible advantages of TDR removal surgery.

The purpose of this study was to compare the clinical midterm results of these two revision strategies for patients with a failed SB Charité III lumbar disc prosthesis. Posterolateral instrumented fusion alone was compared with TDR removal combined with anterior lumbar interbody fusion followed by posterolateral instrumented fusion.

\section{Materials and methods}

\section{Patients}

Ninety patients with a SB Charité III TDR (Waldemar Link, Germany; DePuy Spine, Raynham, MA, USA) were seen in the outpatient clinic. For all these patients TDR implantation had been performed elsewhere. After evaluation, in 39 patients one or more revision surgeries were performed. Indications for revision surgery were recurrent back and leg pain, and the presence of a TDRrelated pathology such as facet joint degeneration, subsidence and migration as observed on plain radiographs, CTscan and/or MRI (Table 1). Adjacent disc degeneration was observed in 13 patients; however, it is uncertain whether this is caused as a consequence of the TDR.

In 21 patients, the TDR was removed and after clearing of periprosthetic fibrous tissue and sclerotic bone, the intervertebral defect was filled with a bone strut graft. In addition, an instrumented posterolateral fusion was performed [16] (removal group). In the 18 other patients, posterolateral instrumented fusion without removal was performed (fusion group). The surgical technique of both surgeries was described in detail by de Maat et al. [16]. Because of persisting pain, in 8 of these 18 patients TDR removal was performed several years later as a second stage revision surgery (range 1.5-7.5 years). For these eight patients, data were available before and after fusion (stage 1) as well as before and after removal of the TDR (stage 2). An overview of the included patients is shown in Fig. 1.

\section{Clinical outcome measurements and complications}

For all patients clinical evaluations were available pre- and post-revision surgery. These evaluations included a 10-point visual analogue scale (VAS) for pain and Oswestry
Table 1 Summary of patient and clinical variables for TDR removal and fusion only group

\begin{tabular}{llll}
\hline & TDR removal $(n=21)$ & Fusion only $(n=18)$ & $P$ value \\
\hline Sex (male:female) & $15: 6$ & $10: 8$ & 0.31 \\
Mean age insertion TDR & 43.4 (range 32-56) & 40.7 (range 30-63) & 0.11 \\
Mean time in situ TDR & 9.1 (range 3.1-16.0) & 7.2 (range 1.7-14.8) & 0.20 \\
Operated levels & & & 0.90 \\
1 level & & & \\
L2-L3 & 0 & 1 & \\
L3-L4 & 2 & 0 & \\
L4-L5 & 10 & 4 & \\
L5-S1 & 4 & 9 & \\
2 level & & 4 & \\
L4-L5, L5-S1 & 4 & & \\
3 level & 1 & 0 & \\
L2-L3, L4-L5,L5-S1 & & 9 & \\
Complications & 8 & 6 & \\
Subsidence & 2 & 14 \\
Migration & 10 & 4 & 1 \\
Facet joint degeneration & 2 & 8 & \\
Breakage metal wire & 5 & & \\
Osteolysis & & & \\
Adjacent disc degeneration & & & \\
\hline
\end{tabular}


Disability Index (ODI, 0-100 points) questionnaire. According to the FDA-criteria, clinical success was defined as a $\geq 25 \%$ improvement in ODI between pre- and postrevision surgery [1]. Similarly for the VAS pain score, a $\geq 25 \%$ improvement was considered as clinically successful. In addition, intra- and post-operative complications of both revision strategies were assessed.

\section{Statistical analysis}

Analyses were performed using SPSS 16. Non parametric tests, i.e. Mann-Whitney and Wilcoxon, were used to test the mean. The mean values were given along \pm standard error of the mean (SEM); $P$ values $<0.05$ were considered statistically significant.

\section{Results}

There were no significant differences between the removal group ( $n=21)$ and fusion group $(n=18)$ with respect to sex, age at insertion of the TDR, mean time in situ of the TDR and number of operated levels (Table 1).

The mean follow-up in the removal group was 3.7 years (range 1.0-6.4 years) and 4.4 years (range $0.7-11.0$ years) in the fusion group $(P=0.82)$.

\section{VAS pain scores}

The mean \pm SEM (standard error of the mean) pre-revision surgery VAS score was $7.9 \pm 0.3$ in the removal group and $7.8 \pm 0.2$ in the fusion group $(P=0.33)$. Postrevision VAS scores were $6.0 \pm 0.4$ and $7.0 \pm 0.4$ in the removal group and in the fusion group, respectively $(P=0.09)$. In both groups, a substantially lower VAS score was observed after revision surgery. Only in the

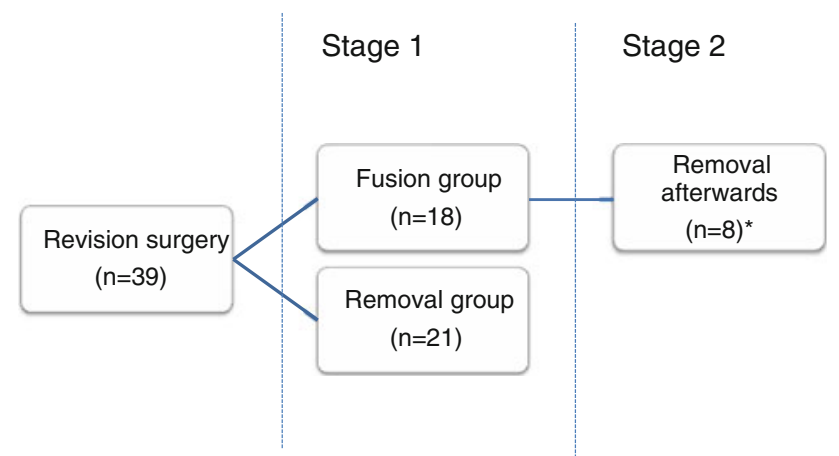

Fig. 1 Overview of the patients who underwent revision surgery after TDR implantation. In these eight patients, pre- as well as postrevision surgery data were available for the fusion and removal revision surgeries (asterisk) removal group, there was a significant decrease in VAS score at post-revision surgery compared to pre-revision surgery $(P<0.01)$ (Fig. 2a).

The percentage of improvement after revision surgery in both groups is shown in Fig. 3a. According to the abovementioned FDA-criteria, in which an improvement of $\geq 25 \%$ was considered to be clinically successful, 10 out of 21 patients $(47.6 \%)$ in the removal group and 4 out of
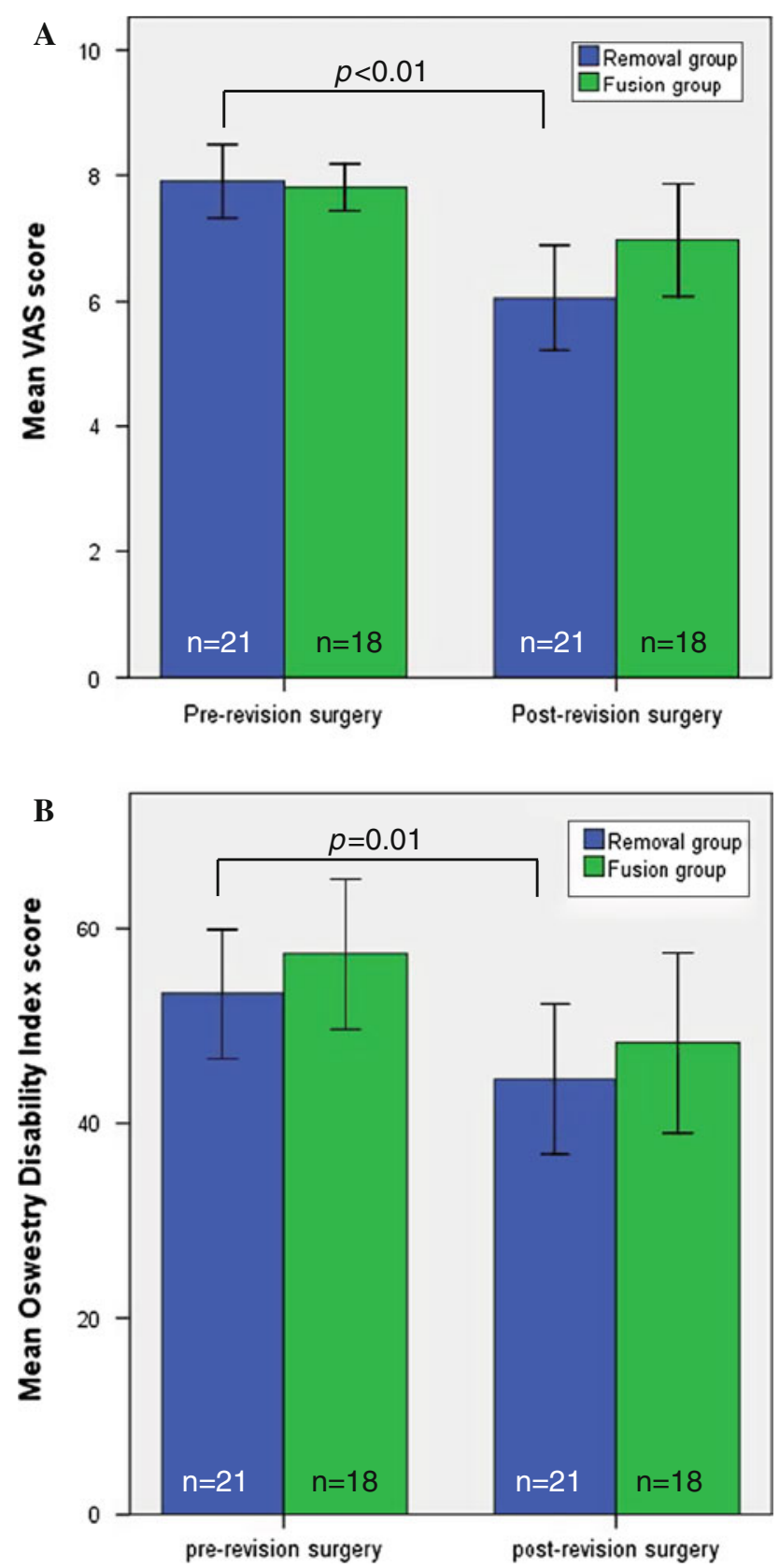

Fig. 2 a Mean VAS scores for both groups pre- and post-revision surgery. b Mean Oswestry Disability Index for both groups during pre- and post-revision surgery. The error bars represent standard error of the mean 
18 patients $(22.2 \%)$ in the fusion group were clinically improved $(P=0.14)$.

\section{Oswestry Disability Index}

The mean pre- and post-revision surgery ODI scores were similar between the removal and fusion groups $(P=0.57$, $P=0.61$, respectively). The ODI in the removal group improved from $53.2( \pm 3.3)$ to $44.5( \pm 3.8)(P=0.01)$, and in the fusion group from $57.3( \pm 3.9)$ to $48.2( \pm 4.6)$ $(P=0.06)$ (Fig. 2b).

The percentage of improvement after revision surgery in both groups is shown in Fig. 3b. A clinically relevant improvement of $\geq 25 \%$ was present in 4 out of 21 patients $(21.1 \%)$ in the removal group and in 5 out of 18 patients $(27.8 \%)$ in the fusion group were clinically improved $(P=0.69)$.
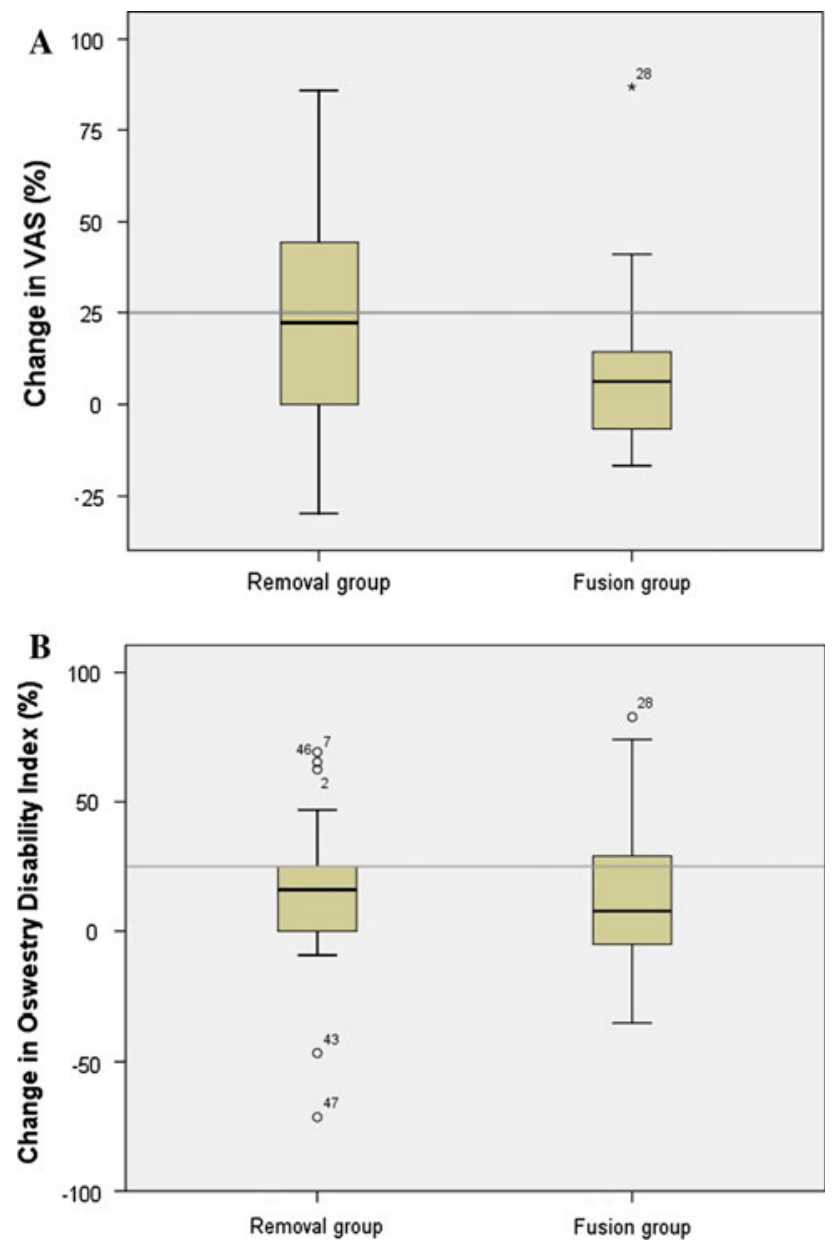

Fig. 3 Box plot with a percentage change in VAS score in both revision strategy groups during pre- and post-revision surgery, b percentage change in ODI score in both revision strategy groups during pre- and post-revision surgery. The line represents a clinical success rate of $25 \%$. The error bars represent the upper and lower quartiles

\section{Second stage revision surgery}

In the fusion group, the eight patients with persisting symptoms who underwent TDR removal at a later timepoint as a second stage revision surgery had a mean followup period of 3.1 years (range 0.7-7.3) after fusion, while the other 10 patients had a mean follow-up of 5.6 years (range 1.8-11.0, $P=0.01$ ). From the abovementioned eight patients, a mean follow-up of 3.1 years (range
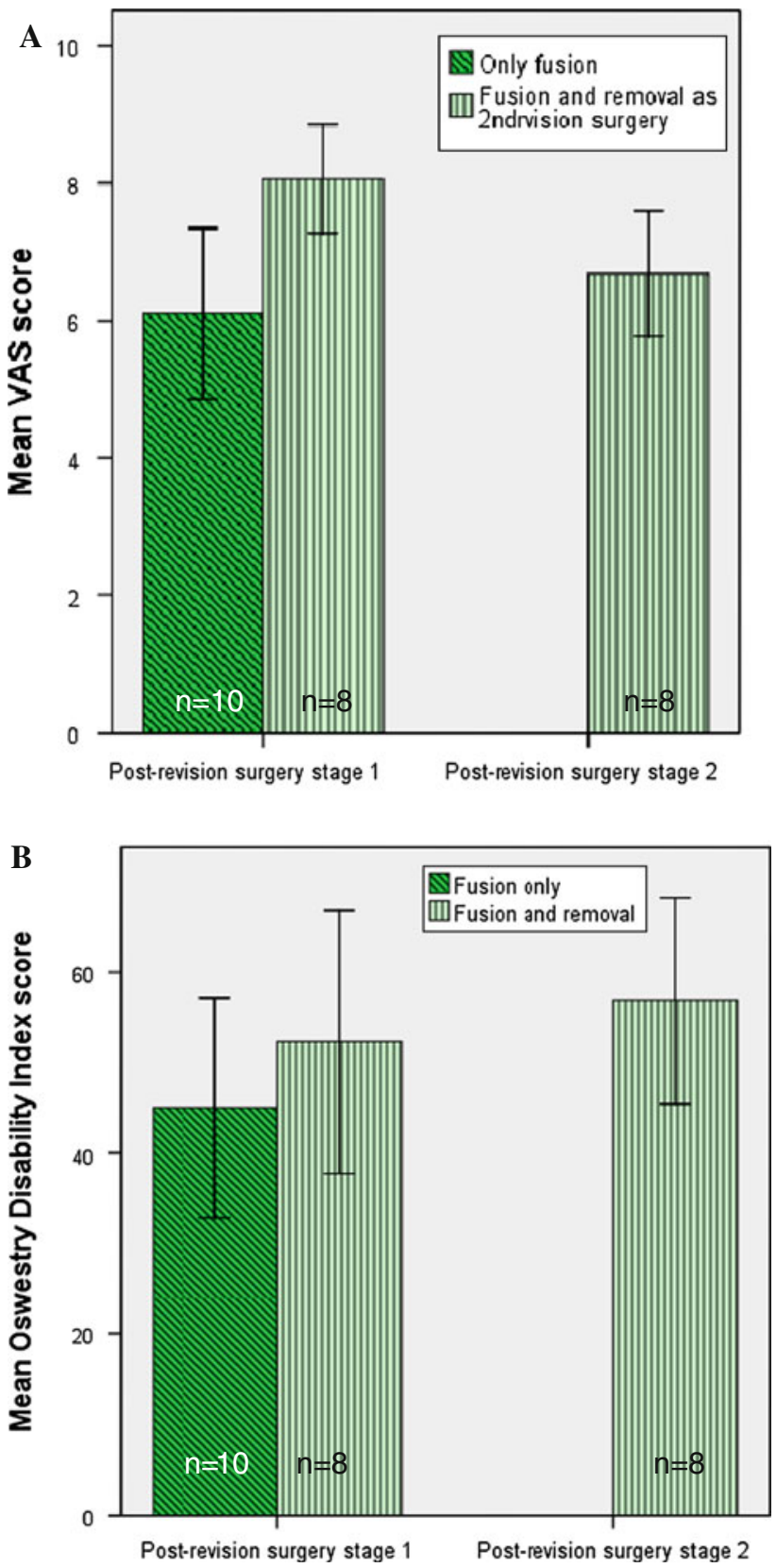

Fig. 4 a Mean VAS scores for the fusion subgroups. b Mean Oswestry Disability Index scores for the fusion subgroups. Of the 18 patients, eight patients underwent TDR removal as a second revision surgery. The error bars represent standard error of the mean 
1.4-5.0) was available after their second stage revision surgery, TDR removal.

In the fusion group, there was a significant difference in post-revision surgery VAS score between the patients who underwent TDR removal at a later time-point $(n=8)$ and those who did not $(n=10)(P<0.01)$. The patient group who underwent removal as a second stage revision surgery $(n=8)$ had a decreased VAS score of $8.1 \pm 0.4$ to $6.7 \pm 0.5$ in time $(P=0.06)$ (Fig. $4 a)$.

Patients in the fusion group who would undergo removal as a second stage revision had a similar ODI score as those who would not ( $P=0.60$ post-revision surgery stage 1$)$. The patient group who underwent removal as a second revision surgery $(n=8)$ had a ODI score of $52.3 \pm 7.3$ and $56.8 \pm 5.7$ after stage 1 and stage 2 revision surgery, respectively (Fig. 4b).

\section{Complications}

An overview of the intra- and post-operative complications from both revision procedures is shown in Table 2. Intraoperatively, no complications were seen in the fusion group, whereas in the removal group different types of vessel bleeding $(n=6)$, a small colon lesion $(n=1)$ and decreased sensitivity of the groyne $(n=2)$ were observed. In one patient TDR removal was planned, however, due to an intraoperative rupture of the small intestine this procedure was abandoned and only posterior fusion was performed. This patient was thus included in the fusion group. In one patient a lesion of the ureter occurred intra-operatively which necessitated resection of the left kidney at a second stage. In the fusion only group two patients developed a pseudo-arthrosis.

\section{Discussion}

The clinical results of two revision strategies for failed TDR's were studied after a mean follow-up of 3.7 and
4.4 years in the removal and fusion group, respectively. In 18 patients, a posterolateral instrumented fusion without removal was performed and in 21 patients removal of the TDR was combined with anterior interbody fusion followed by posterolateral instrumented fusion. The mid-term VAS and ODI scores significantly improved in the removal group compared to pre-revision surgery, while no significant improvement was found in the fusion group. However, the VAS and ODI scores were comparable for both groups at both time points. A clinical successful improvement ( $\geq 25 \%$ ) in VAS was found in $47 \%$ in the removal group and in $22 \%$ in the fusion group. For ODI, $21 \%$ in the removal group and $27 \%$ in the fusion group showed a clinical successful improvement. An important point to consider is that, in contrast to the fusion only group with no intra-operative complications, the TDR removal group showed substantial complications in nine patients $(31 \%)$ during surgery.

The present study was limited by the relatively small number of cases in both groups which may have induced a type II error. In addition, both surgical groups showed heterogeneous patient characteristics. For example, the number of patients who underwent a second stage revision surgery varies considerably.

In the literature, a wide range of complications has been reported in TDR implantation studies. These complications can be divided into (1) treatment related (e.g. pain, wound problems), (2) anterior approach related (e.g. vascular injury, retrograde ejaculation) and (3) prosthesis related (e.g. subsidence, migration) [11]. The number of reoperations varied between 2.3 and $14 \%$ [1, 6, 7, 11, 12, 17]. McAfee et al. [6] reported on 24 patients (9\%) who underwent an anterior TDR revision surgery. Those patients who underwent a revision for failed TDR, all had a suboptimal or poor placement of the TDR. The mean time to revision was 9 months (range 3 days to 34 months). In 4 of these 24 patients (16.7\%), a vascular injury was encountered [6]. In another study, from Leary et al. [17], 18
Table 2 Intra- and postoperative complications resulting from both revision strategies

\begin{tabular}{lll}
\hline & TDR removal $(n=21)$ & Fusion only $(n=18)$ \\
\hline Intra-operative & Left common iliac artery lesion $(n=1)$ & \\
& Left common iliac vein lesions $(n=2)$, one & \\
& resulting & \\
& in a deep venous thrombosis of the left leg & \\
& Bleeding ascending lumbar vein $(n=1)$ & \\
& Pronounced bleeding intervertebral defect $(n=1)$ & \\
& Major blood loss $(5,100$ cc $)(n=1)$ & \\
& Small colon lesion $(n=1)$ & \\
& Decreased sensitivity in the left groyne $(n=2)$ & Pseudo-arthrosis \\
Post-operative: & Resection left kidney after ureter lesion $(n=1)$ & $(n=2)$ \\
$0-3$ years & & \\
\hline
\end{tabular}


patients underwent an anterior revision procedure after an average follow-up of 6 months (range 9 days to 4 years). No significant vascular, ureteral and neurological injuries were encountered. However, two patients had a minor left iliac vein injury $(11.1 \%)$, and one case of retrograde ejaculation was seen that resolved spontaneously [17]. In our patient group we encountered a vascular injury in 6 out of $29(20.7 \%)$. To avoid a ureter lesion, we suggest to insert a J-catheter in the left ureter if a left retroperitoneal approach is used. When considering revision surgery it should be realized that removal of the prosthesis has increased risks because of vascular structures and scar tissue [16-18]. The assistance of a vascular surgeon during TDR removal surgery is strongly recommended [16, 17]. Furthermore, the time between TDR implantation and revision may be of importance. In our patients, TDR removal was performed at much larger follow-up as compared to the previously mentioned studies (mean 9.4 years, range 3.1-16.3). When TDR removal is indicated, it should be performed as soon as possible after the initial TDR implantation because of the development of scar tissue and adhesions [17]. Complications such as wound infection and injury to the (superior) hypogastric plexus, which may induce erectile dysfunction and retrograde ejaculation, were not encountered in our series [19].

There is an ongoing discussion about the optimal revision strategy for failed TDRs. In case of an acceptable implant status and position, posterior fusion can be addressed for the treatment of recurrent back pain thought to be facet joint in origin. When the TDR has subsided, migrated or mechanically failed, the pain can be addressed by TDR removal. In our own experience, the results of posterolateral fusion without TDR removal were disappointing in most patients. Therefore, we combined fusion with TDR removal in all cases if the patient accepted the risks of retrieval surgery.

In a previous study, we studied periprosthetic fibrous tissues of the first 16 patients with TDR removal using light microscopy [13]. Results of that study demonstrated the presence of polyethylene wear particles and of periprosthetic inflammatory reactions around a failed TDR in 15 out of 16 patients [13]. We therefore hypothesized that TDR removal will reduce back and leg pain in failed TDRs because the source of wear debris generation is removed, which may diminish inflammatory mediated pain. The present study showed that, although there was no significant difference between the removal and fusion group during post-revision surgery, the VAS score improved significantly in the removal group after 3.1 years. Removal surgery as a second revision strategy in patients who still experience a high amount of pain after posterolateral fusion reduced VAS pain scores nonsignificantly.
The aim of the present study was to provide mid-term clinical follow-up data on two TDR revision strategies. In agreement with our previous study [15], the VAS and ODI scores showed similar wide ranges, which indicate substantial variability in outcome between the individual patients. Possible explanations for these wide intervals are patient-related factors like number of previous surgeries and number of affected levels. Larger groups will be necessary to assess the effect of patient factors on the success rate of a revision surgery.

In conclusion, the benefit of removing the prosthesis after failed TDR remains unclear. Removal of the TDR may be justified. However, the patient should be counselled about potential risks and complications of this kind of revision surgery which should be carefully weighed up against the possible benefits of TDR removal.

Conflict of interest None of the authors has any potential conflict of interest.

Open Access This article is distributed under the terms of the Creative Commons Attribution License which permits any use, distribution, and reproduction in any medium, provided the original author(s) and the source are credited.

\section{References}

1. Blumenthal S, McAfee PC, Guyer RD, Hochschuler SH, Geisler FH, Holt RT, Garcia R, Jr., Regan JJ, Ohnmeiss DD (2005) A prospective, randomized, multicenter Food and Drug Administration investigational device exemptions study of lumbar total disc replacement with the CHARITE artificial disc versus lumbar fusion: part I-evaluation of clinical outcomes. Spine 30 (14):1565-1575 (discussion E1387-E1591)

2. McAfee PC, Cunningham B, Holsapple G, Adams K, Blumenthal S, Guyer RD, Dmietriev A, Maxwell JH, Regan JJ, Isaza J (2005) A prospective, randomized, multicenter Food and Drug Administration investigational device exemption study of lumbar total disc replacement with the CHARITE artificial disc versus lumbar fusion: part II-evaluation of radiographic outcomes and correlation of surgical technique accuracy with clinical outcomes. Spine 30(14):1576-1583 (discussion E1388-E1590)

3. de Kleuver M, Oner FC, Jacobs WC (2003) Total disc replacement for chronic low back pain: background and a systematic review of the literature. Eur Spine J 12(2):108-116

4. Geisler FH, Guyer RD, Blumenthal SL, McAfee PC, Cappuccino A, Bitan F, Regan JJ (2008) Effect of previous surgery on clinical outcome following 1-level lumbar arthroplasty. J Neurosurg 8(2):108-114

5. Lemaire JP, Carrier H, el Sariali H, Skalli W, Lavaste F (2005) Clinical and radiological outcomes with the Charite artificial disc: a 10-year minimum follow-up. J Spinal Disord Tech 18(4):353-359

6. McAfee PC, Geisler FH, Saiedy SS, Moore SV, Regan JJ, Guyer RD, Blumenthal SL, Fedder IL, Tortolani PJ, Cunningham B (2006) Revisability of the CHARITE artificial disc replacement: analysis of 688 patients enrolled in the U.S. IDE study of the CHARITE artificial disc. Spine 31(11):1217-1226

7. David T (2007) Long-term results of one-level lumbar arthroplasty: minimum 10-year follow-up of the CHARITE artificial disc in 106 patients. Spine 32(6):661-666 
8. Guyer RD, McAfee PC, Banco RJ, Bitan FD, Cappuccino A, Geisler FH, Hochschuler SH, Holt RT, Jenis LG, Majd ME, Regan JJ, Tromanhauser SG, Wong DC, Blumenthal SL (2009) Prospective, randomized, multicenter Food and Drug Administration investigational device exemption study of lumbar total disc replacement with the CHARITE artificial disc versus lumbar fusion: five-year follow-up. Spine J 9(5):374-386

9. Guyer RD, McAfee PC, Hochschuler SH, Blumenthal SL, Fedder IL, Ohnmeiss DD, Cunningham BW (2004) Prospective randomized study of the Charite artificial disc: data from two investigational centers. Spine J 4(6 Suppl):252S-259S

10. Putzier M, Funk JF, Schneider SV, Gross C, Tohtz SW, Khodadadyan-Klostermann C, Perka C, Kandziora F (2006) Charite total disc replacement—clinical and radiographical results after an average follow-up of 17 years. Eur Spine J 15(2):183-195

11. van den Eerenbeemt KD, Ostelo RW, van Royen BJ, Peul WC, van Tulder MW (2010) Total disc replacement surgery for symptomatic degenerative lumbar disc disease: a systematic review of the literature. Eur Spine J 19(8):1262-1280

12. Yajun W, Yue Z, Xiuxin H, Cui C (2010) A meta-analysis of artificial total disc replacement versus fusion for lumbar degenerative disc disease. Eur Spine J 19(8):1250-1261

13. Punt IM, Cleutjens JP, de Bruin T, Willems PC, Kurtz SM, van Rhijn LW, Schurink GW, van Ooij A (2009) Periprosthetic tissue reactions observed at revision of total intervertebral disc arthroplasty. Biomaterials 30(11):2079-2084

14. Cunningham BW, Hu N, Beatson HJ, Serhan H, Sefter JC, McAfee PC (2009) Revision strategies for single- and two-level total disc arthroplasty procedures: a biomechanical perspective. Spine J 9(9):735-743

15. Punt IM, Visser VM, van Rhijn LW, Kurtz SM, Antonis J, Schurink GW, van Ooij A (2008) Complications and reoperations of the SB Charite lumbar disc prosthesis: experience in 75 patients. Eur Spine J 17(1):36-43

16. de Maat GH, Punt IM, van Rhijn LW, Schurink GW, van Ooij A (2009) Removal of the Charite lumbar artificial disc prosthesis: surgical technique. J Spinal Disord Tech 22(5):334-339

17. Leary SP, Regan JJ, Lanman TH, Wagner WH (2007) Revision and explantation strategies involving the CHARITE lumbar artificial disc replacement. Spine 32(9):1001-1011

18. Spivak JM, Petrizzo AM (2010) Revision of a lumbar disc arthroplasty following late infection. Eur Spine J 19(5):677-681

19. Tiusanen H, Seitsalo S, Osterman K, Soini J (1995) Retrograde ejaculation after anterior interbody lumbar fusion. Eur Spine $\mathbf{J}$ 4(6):339-342 